\title{
Future Skills - Leitmarken einer neuen Bildungskonzeption für Hochschulen
}

\section{\#hinführung}

Future Skills hat in der öffentlichen Diskussion über Hochschulbildungskonzepte mittlerweile zu einem entscheidenden Wandel beigetragen, den wir hier als Future Skills Turn bezeichnen. Diesen Aufzuarbeiten und in seiner Tragweite für die Konzeption künftiger Hochschulbildung zu erfassen, ist der Gegenstand dieses Buches. Als Begriff hat Future Skills einen Einfluss gewonnen, wie er in den siebziger Jahren des letzten Jahrhunderts von Begriffen wie Chancengleichheit oder Wissenschaftsorientierung ausgegangen ist. Solche Leitmarken treten nicht als exakt zugeschnittene und empirisch operationalisierte Konzepte auf, sondern viel eher als begriffliche Verdichtungen breit gefächerter Bündel von Argumenten und Zielsetzungen.

Ausgangspunkt für die enorme Karriere des Konzeptes der Future Skills ist die Diagnose, dass derzeitige Konzepte der Hochschulbildung den drängenden Herausforderungen unserer Gesellschaften keine überzeugenden Zukunftskonzepte entgegenstellen. Weder der nachhaltigen Gestaltung unserer Umwelt noch den damit zusammenhängenden sozialen oder ökonomischen. Während die gesellschaftlichen Problemlagen von einem sich stetig beschleunigenden Globalisierungsprozess und einem immer schneller werdenden digitalen Fortschritt verschärft werden, sind 
genau dieses auch die Kräfte der Ermöglichung für eine Vielzahl neuer Optionen für die menschliche Entwicklung. In dieser Situation digitaler Beschleunigung ist das kennzeichnende Merkmal das der Unsicherheit und die unausweichliche Notwendigkeit die der Gestaltungsverantwortung. Denn die Zukunft ist unvorhersehbar und wir können sie nicht prognostizieren, müssen aber bereit sein, sie zu gestalten.

Kinder, die im nächsten Jahr in die Grundschulen kommen, werden in zehn bis zwölf Jahren in eine Berufsausbildung oder ein Studium gehen und in fünfzehn Jahren diejenigen sein, die als junge Berufstätige beginnen, unsere Gesellschaft zu prägen. Über diese Zukunft wissen wir wenig. Im Jahr 2060-2065 werden sie aller Voraussicht nach ihrer Erwerbstätigkeit beenden. Über diese Zukunft wissen wir nichts. Unsere Schulen müssen sie auf Jobs vorbereiten, die es heute noch nicht gibt, auf Technologien, Apps und Anwendungen, die heute noch nicht erfunden worden sind, darauf, in einer Gesellschaft zu leben, deren Strukturen wir heute nicht absehen können, und darauf, mit Herausforderungen umzugehen, die heute noch nicht erkennbar sind. Es ist unser aller gemeinsame Verantwortung, das Beste aus den Möglichkeiten zu machen und Wege zu finden, mit dieser ungewissen Zukunft umzugehen. Dabei geht es um nicht mehr und nicht weniger als den Erhalt unseres Planeten und unserer Lebensgrundlagen.

Das Lösen der gesellschaftlichen Problemlagen, wie sie etwa mit dem Klimawandel verbunden sind, der Herausforderungen der zukünftig noch zunehmenden Migration, der Konflikte, die durch populistische Gesellschafts- und Politikentwürfe entstehen und der damit verbundene Frage nach der Zukunft der Demokratie - all dies erfordert die Fähigkeit, neue und bisher unbekannte Ansätze zu entwickeln, neue Wege zu gehen und bislang Unverbundenes auf neue Weise miteinander in Beziehung zu setzen. In der Bildung und Wissenschaft wird dies nur dann gelingen, wenn wir im besten Sinne inter- und transdisziplinär daran arbeiten, die Lösungsbeiträge einer jeden Disziplin und Wissenschaft zusammenzutragen, kritisch zu reflektieren und aufeinander zu beziehen. Hochschulen tun sich dabei schwer - denn sie alle teilen ein gemeinsames Handicap: Die Geschichte der Wissenschaft, Forschung und damit auch der Hochschulbildung ist eine Geschichte der Differenzierung, Spezialisierung und Abgrenzung der Disziplinen - die fast 18.000 Studiengänge, die an deutschen Hochschulen angeboten werden, zeugen davon. Die Institution Hochschule steht vor der Herausforderung, sich selber neu erfinden zu müssen - und das in einer Zeit, in der sie sich in einem enormen Wachstumsprozess befindet und weltweit eine Quote von 70 Prozent Studierenden einer Alterskohorte oder mehr bis ins Jahr 2050 prognostiziert wird. Das ist in etwa so, als müsse man bei einem Autorennen, mitten in der Steilkurve und während eines gefährlichen Überholmanövers die Pilotin/ den Piloten wechseln. 


\section{\#futureskills}

Das Forschungsprojekt NextSkills zielt darauf ab, im Rahmen eines multimethodischen Forschungsdesigns und über internationale Konsultationen Modelle und Beschreibungen für zukünftig relevante Fähigkeiten, sogenannte Future Skills, zu finden. ${ }^{1}$ Dabei sollen Future Skills diejenigen Fähigkeiten sein, die es Hochschulabsolventinnen und -absolventen ermöglichen, die Herausforderungen der Zukunft bestmöglich zu meistern. In den Ergebnissen zeigt sich: Um mit den zukünftigen Herausforderungen umzugehen, müssen Studierende Neugier entwickeln, Vorstellungskraft, Visionsfähigkeit, Resilienz und Selbstbewusstsein sowie die Fähigkeit, selbstorganisiert zu handeln. Sie müssen in der Lage sein, die Ideen, die Perspektiven und die Werte anderer zu verstehen und zu respektieren und sie müssen mit Fehlern und Rückschritten umgehen können und gleichzeitig achtsam voranschreiten, auch gegen Schwierigkeiten.

In zahlreichen Gesprächen, Interviews und Analysen wurde uns deutlich, dass Future Skills auch darauf abzielen müssen, Bewusstsein für lokale und globale Herausforderungen zu befördern; Bewusstsein und Achtsamkeit dafür zu erlangen, wie sich der Klimawandel auf die Natur und Umwelt auswirkt und wie Studierende Fähigkeiten erlangen können, in gesellschaftlichen Zusammenhängen mitzuwirken, um diese Auswirkungen zu reduzieren oder umzukehren. Es geht auch darum, gesellschaftliche Themen wie beispielsweise den demographischen Wandel oder Migrationsherausforderungen zu gestalten.

Future Skills zu fördern bedeutet auch, ein Bildungssystem zu gestalten, welches die zukünftigen Bürgerinnen und Bürger in die Lage versetzt, mit damit verbundenen Herausforderungen umzugehen und in der Gesellschaft für Kohärenz zu sorgen, Offenheit, Toleranz, ein Bewusstsein für Unterschiedlichkeit wertzuschätzen und gerade nicht, populistischen Erklärungen zu erliegen. Es wurde uns deutlich, dass die Frage, wie junge Leute für die Teilhabe an gesellschaftlichen Systemen und Prozessen befähigt werden, und wie wir Frieden, Bewahrung der Schöpfung und Gemeinschaft als Werte in einer zukünftigen Gesellschaft stärken können, zukünftig über die Relevanz unserer Hochschulen entscheiden.

Dabei wird das heutige Fach- und Expert(inn)enwissen nur noch einen kleinen Teil darstellen, an dem sich zukünftige Generationen auf ihrer Suche nach Lösungen komplexer Probleme orientieren werden können. Sie werden von mehr angetrieben werden als von Karriere, einem guten Job und einem hohen Einkommen. Auch um das Wohl ihrer Freunde und Familien, ihrer Communities und des Planeten als Ganzem, werden sie sich bemühen müssen. Mitgefühl, Achtsamkeit und Leiden-

1 Mehr zum NextSkills Projekt siehe hier: http://www.NextSkills.org 
schaft werden zu expliziten Bildungszielen der Hochschulen der Zukunft werden. Es wird darum gehen, Bildungskonzepte einzusetzen, die Lernende mit Kraft, Energie und Überzeugung ausstatten und mit der Fähigkeit, diese wertschätzend zu kommunizieren. Die Kompetenzen, die sie brauchen, müssen sie in die Lage versetzen, ihr eigenes Leben zu gestalten und zum guten Leben anderer beizutragen.

Hochschulen tun gut daran, sich vom Ziel abzuwenden, Wissensbestände zu vermitteln, in denen es primär um in sich geschlossene und gut prüfbare Zusammenhänge geht, für die es richtige und falsche Antworten gibt. Zukünftig wird es darauf ankommen, anhand von Fragestellungen zu lernen, für die es keine unmittelbar richtigen Antworten gibt, sondern bei denen es darum geht abzuwägen, plausibel zu argumentieren und das Werthaltungen zu vertreten. Um herauszufinden, welche Fähigkeiten dies sind und wie diese am besten entwickelt werden können, wurde das NextSkills Projekt ins Leben gerufen. Das Ziel dieses Projektes ist es, Hochschulen, ihren Leitungen und ihren Lehrenden Antworten dafür zu liefern, in welche Richtung Bildungsziele, Strukturen und Prozesse gestaltet werden müssen. Im Zentrum stehen 3 Fragen:

1. Welche Fähigkeiten brauchen Menschen in der Zukunft, um ihre Welt und Umwelt als Bürgerinnen und Bürger in einer globalisierten Welt zu gestalten? Welche Fähigkeiten brauchen Mitarbeiterinnen und Mitarbeiter, um die ständige Weiterentwicklung und stetige Anpassung an neue Situationen in Organisationen und im Arbeitsleben zu bewältigen? Diese Fähigkeiten nennen wir Future Skills.

2. Wie können Organisationen ihre Mitarbeiterinnen und Mitarbeiter dabei unterstützen, diese Fähigkeiten zu erlangen und welche Organisationsformen und -strukturen werden benötigt, um die dafür optimalen Organisationskulturen herauszubilden?

3. Was können Hochschulen tun, um diese Fähigkeiten bei Studierenden zu fördern? Wie müssen Studium und Lehre gestaltet sein und welche hochschuldidaktischen Formen eignen sich?

In diesem Buch beschreiben wir die Ergebnisse dieser Arbeit. Die vorgestellten Konzeptionen sind durch Tiefeninterviews, Expertenbeurteilungen und internationale Delphi-Studien abgesichert.

Stellt man Future Skills in den Mittelpunkt der Überlegungen für Hochschulbildung, dann zeigt sich an vielen Punkten die Notwendigkeit, die Hochschule als Ort des Forschens, Lehrens und Lernens neu zu denken. Dabei gilt: All das, was leicht $\mathrm{zu}$ unterrichten ist und leicht geprüft werden kann, ist auch leicht zu digitalisieren - und damit auch zu automatisieren. Future Skills wie Kreativität, Selbstkompetenz, Reflexionskompetenz oder Design Thinking-Kompetenz benötigen jedoch 
ausgeklügelte Vermittlungsformen. Es geht also darum, die Frage zu stellen, wie die Förderung von Future Skills in den Hochschulcurricula verankert werden kann. Dabei geht es um eine Konzentration auf aktive, gestaltende Lehr- und Lernformen und Bildungsziele, die komplexe Prüfungsszenarien benötigen und die über reine Wissensvermittlung hinausgehen und Kompetenzen in den Vordergrund stellen.

\section{\#bisher}

Die NextSkills Studie findet nicht in einem Vakuum statt. Die Frage, welches die Fähigkeiten sein sollen, die in einem Bildungssystem für zukünftige Generationen den Lernkonzepten zugrunde liegen, ist hoch relevant - und eine bereits vielschichtig diskutierte. Nachdem in den achtziger und neunziger Jahren die Forschung zu Graduate Attributes im Vordergrund stand, gibt es derzeit eine regelrechte Renaissance an wissenschaftlichen Arbeiten zu diesem Thema. Das sind zum einen Arbeitsmarktstudien, die sich die Frage stellen wie die Zukunft der Arbeit aussieht, bei denen Digitalisierung einen großen Einfluss hat. Das sind zweitens Studien der Gesellschaft, die sich die Frage stellen, wie die Gesellschaft im Jahre 2030 oder 2050 aussehen wird. Das sind Fragen wie: Wird Arbeit weiterhin das sinnstiftende Element in unserem Leben sein? Was sind die Risiken, die Individuen in einer Gesellschaft zu bewältigen haben und was sind Strategien zur Bewältigung selbiger? Auch dabei spielt die digitale Durchdringung der gesamten Privatsphäre eine große Rolle. Und es ergeben sich drittens Fragen in Bezug auf Bildungskonzeptionen, die sich damit auseinandersetzen wie Studierende auf unvorhersagbare Zukünfte vorbereitet werden können. Viertens und letztens sind es viele Ansätze, die im Bereich internationaler Organisationen wie der Europäischen Union (EU), der Organisation for Economic Co-operation and Development (OECD) oder der United Nations Educational, Scientific and Cultural Organization (UNESCO) entstehen, die die Frage stellen, wie Gesellschaften zusammen in einer Weise lernen und leben können, sodass die globalen Herausforderungen angemessen und wirksam bearbeitet werden können. All diese unterschiedlichen Perspektiven die seit etwa den neunziger Jahren zunehmend stärker diskutiert werden, rücken in den Fokus durch internationale Zusammenarbeit, globale Vernetzung und Digitalisierung. Die Diskussionen schlagen sich in Konzepten wie zum Beispiel den Sustainable Development Goals (SDG) oder anderen, grenzüberschreitenden Bildungs- und Gesellschaftsentwürfen nieder.

Die Frage welche Fähigkeiten junge Menschen der künftigen Generationen benötigen, um mit den beschriebenen Herausforderungen umzugehen, hat also 
große Konjunktur. Sie wird viel diskutiert und ist derzeit eines der heißen Themen, nicht nur in der Bildungswissenschaft, sondern auch in den Wirtschafts- und Arbeitswissenschaften. Die meisten der Ansätze gehen dabei empirisch analytisch vor und versuchen, durch die Analyse bestehender Entwicklung die Zukunft zu prognostizieren, indem sie beispielsweise die Entwicklung von neuen Berufen und Berufsfeldern zugrunde legen oder die Geschwindigkeit der Technologieentwicklung und ihrer Anwendung auf die Automatisierung in Arbeitsprozessen (linear) fortschreiben und so zu neuen Professionsprofilen kommen. Aus denen heraus werden wiederum Qualifikationsanforderungen abgeleitet, die dann in Schulen und Hochschulen zu Kompetenzprofilen entwickelt werden.

\section{\#emergenz}

Dieser Ansatz hat Grenzen, die nun immer absehbarer werden. Es wird deutlich, dass die Debatte um die Zukunftsfähigkeiten, die benötigt werden, um die gesellschaftlichen Herausforderungen derzeitiger und zukünftiger Generationen zu bewältigen, vor allem eines berücksichtigen muss: Einen sich immer schneller und nicht-linear wandelnden Handlungskontext. Dieses Charakteristikum hält in immer mehr Bildungskonzepte Einzug - zunächst auf einer rein deskriptiven Ebene. Es wird gefragt, wie damit umzugehen sei, wenn Studierende nicht mehr auf ein bestimmtes Ziel hin vorbereitet werden können, weil ein klar definierbares Wissensziel an sich nicht mehr bekannt ist. In jüngster Zeit kommen systematischere, wissenschaftliche Entwürfe hinzu, in denen die Frage der Unvorhersehbarkeit auf wissenschaftlich-theoretischer Ebene diskutiert wird. Hierbei sind derzeit die Entwürfe aus der Ökosystemtheorie, aus der Physik im Bereich der Forschung über emergente Systeme und Selbstorganisation sowie die Kybernetik in der Verhaltensforschung und Biologie zu nennen. Ihnen liegt die Erkenntnis zugrunde, dass Entwicklungen in Systemen oft zu neuen Zuständen führen, die sich aus den vorherigen Zuständen nicht ableiten lassen. Die so bezeichnete emergente Entwicklung hat das Kennzeichen der Irreduzibilität, also der Unmöglichkeit des linearen Fortschreibens einer Entwicklung in eine zukünftige Entwicklung, da sich die zukünftige Entwicklung, als neuer Status nicht mehr auf den vorherigen reduzieren lässt; und zweitens das Kennzeichen der Unvorhersagbarkeit, also der Unfähigkeit des Prognostizierens des nächsten, nachfolgenden Zustandes. Übertragen auf gesellschaftliche Prozesse, politische Prozesse und wirtschaftliche Prozesse, sowie Prozesse der Kommunikation bedeutet dies, dass Individuen künftig mit Situationen umgehen werden müssen, die sich weder vorhersagen noch berechnen lassen. 
Die Frage ist, wie man mit dem Unvorhergesehenem umgeht. Beiträge zu Future Skills müssen Antworten auf diese Frage liefern. Derzeit gibt es nur wenige konkurrierende Ansätze, die sich dieser Frage wirklich systematisch stellen und über einen reinen Kompetenzbegriff hinausgehen. Mit diesem Buch streben wir an, diese Lücke zu schließen. In der vorliegenden Arbeit wird ein Modell für Future Skills entworfen. Es kombiniert bildungstheoretische Aspekte mit Kompetenzkonzepten und Konzeptionen der Selbstorganisation.

\section{\#triplehelix}

Dies ist ein Buch von Übermorgen. Unsere derzeitigen Hochschulbildungskonzepte sind noch stark an Wissensvermittlung orientiert. Wissen aufzubauen und anzuhäufen, um dieses dann in den künftigen beruflichen Handlungssituationen abzurufen, ist - pointiert ausgedrückt - das aktuelle Spiel von Hochschulbildung, Studium und Arbeitsmarkt. Doch es bröckeln die Ränder dieser Entwicklung. Unsere Studie zeigt, dass insbesondere in solchen Arbeitsfeldern, die als hoch agil und gleichzeitig wissensintensiv gelten können, zunehmend nicht mehr abrufbares Wissen die Währung zukünftigen Arbeitsmarkterfolges ist, sondern Future Skills. Im Rahmen der NextSkills Studie wird dazu das Triple Helix-Modell der Handlungsfähigkeit in emergenten Kontexten entwickelt und in Kapitel A2 vorgestellt. Das Triple Helix-Modell zeigt, wie die Wandlungsprozesse mit neuen Fähigkeitsanforderungen zusammenhängen.

Dabei sehen wir das Hochschulsystem weltweit inmitten eines Transformationsprozesses. Alle industrialisierten Gesellschaften stehen strukturell gesehen am Rande einer Bildungsgesellschaft, in der der Druck zur akademischen Bildung stetig steigt. Das geht einher mit einer durch Verfügbarkeit von Kommunikationsmedien immer stärkeren Vernetzung und einer daraus resultierende Konkurrenz global verfügbaren Wissens. Die sich daraus ergebende Beschleunigung der Entwicklung und des Verfalls von Wissensbeständen macht eine ständige lebenslange Aktualisierung des Wissens des Einzelnen notwendig. Wissen ist jedoch nicht genug, es bildet lediglich die Basis für Future Skills. Während die Skills dabei zwar nicht immer neu sind, so gibt ihnen jedoch das Ausmaß, in dem die Performanz in Future Organisations von ihnen abhängt, eine gänzlich neue Relevanz.

Future Skills ist ein schillernder Begriff, dem vielfältige Verständnisse zugrunde liegen. Die bestehenden Ansätze erschöpfen sich dabei oftmals in einer Aneinanderreihung von Begriffselementen, die für Future Skills wichtig sein könnten. Liste reiht sich an Liste. Über diese rein additive Sichtweise hinaus gibt es derzeit lediglich die 
altbekannten aber vielfach nicht umgesetzten kompetenzorientierten Lernszenarien. Dabei wird oft betont, dass es letztlich der Bildungswissenschaft schon immer um Future Skills ging. Denn was, wenn nicht zukünftige Handlungsfähigkeit sollen Bildungskonzepte eigentlich zum Inhalt haben? Future Skills ist damit zugegebener Maßen ein schillernder und populärer Begriff, der aus bildungswissenschaftlicher Sichtweise sicher bereits Konstruktionsdefizite in sich trägt.

Lässt man einmal diese - zugeben gerechtfertigten Defizite - beiseite, so birgt er aber auch Chancen. Er hebt sich ab von der schon etwas eingefahrenen Debatte um die Einführung von Kompetenzen als Konstrukte aus Wissen, Fähigkeiten und Einstellungen, bezieht sich zunächst nicht vordergründig sofort auf die Diskussion um Schlüsselqualifikationen und Kompetenzen und ist international anschlussfähig. Der Begriff ist also reizvoll, bedarf aber einer gehörigen Portion begrifflicher Schärfung und Abgrenzung.

\section{\#aufbau}

Mit dem Buch verfolgen wir zwei Hauptzielsetzungen: Zum einen wollen wir ausführlich über die Ergebnisse des dreijährigen NextSkills Projekts und dessen Gehalt für die deutsch- und englischsprachige Forschung zum Thema Future Skills berichten. Zum anderen geht es uns darum, mit dem Buch einen theoretischen Bezugsrahmen für Future Skills in der Hochschulbildung zu legen und bestehende Forschungen mit Bezug zum Thema darin einzuordnen. Die nachfolgend abgebildete Infografik (Abbildung 1) illustriert die dazu konzipierte Struktur des Buches.

Das Buch ist in drei große Teile gegliedert, denen ein einführendes Kapitel zum Future Skills Turn vorangestellt ist (Kapitel II). Dieser Future Skills Turn wird anhand vielfältiger Beispiele beschrieben, die die zunehmende Bedeutung von Future Skills als künftige Leitorientierung für Hochschulbildung aufzeigen. Dabei geht es nicht etwa um einen neuen Bildungs- oder Kompetenzbegriff, sondern um die Beschreibung derjenigen Kompetenzprofile, die als Future Skills unter Bedingungen von Future Organisations Bedeutung erlangen.

Teil A widmet sich den Future Skills. Dazu wird in Kapitel A 1 zunächst das Studiendesign der NextSkills Studie beschrieben. In Kapitel A 2 wird ein grundlegender theoretischer Rahmen für Future Skills als Bildungskonzeption entwickelt. Dabei wird das sogenannte Triple Helix-Modell der Handlungsfähigkeit in emergenten Praxiskontexten entwickelt. Dem Modell liegen drei Shifts zugrunde, drei wesentliche Änderungen in der Grundstruktur der Arbeitswelt, auf denen das Future Skill Konzept aufbaut. Innerhalb der Forschung zu Future Skills bietet die NextSkills 
Studie mit dem Triple Helix-Modell für Future Skills als erste Studie überhaupt einen theoretischen Bezugsrahmen für Future Skills. Im weiteren Verlauf werden in Kapitel A 3 die siebzehn Future Skills Profile herausgearbeitet, definiert und beschrieben. Kapitel A 4 analysiert die Ergebnisse der internationalen NextSkills Delphi-Studie in Bezug auf den Reifegrad derzeitiger Hochschulbildung und deren Fähigkeit, die Entwicklung von Future Skills bei Studierenden zu unterstützen.

\section{Future Skills Turn}

Kap. II

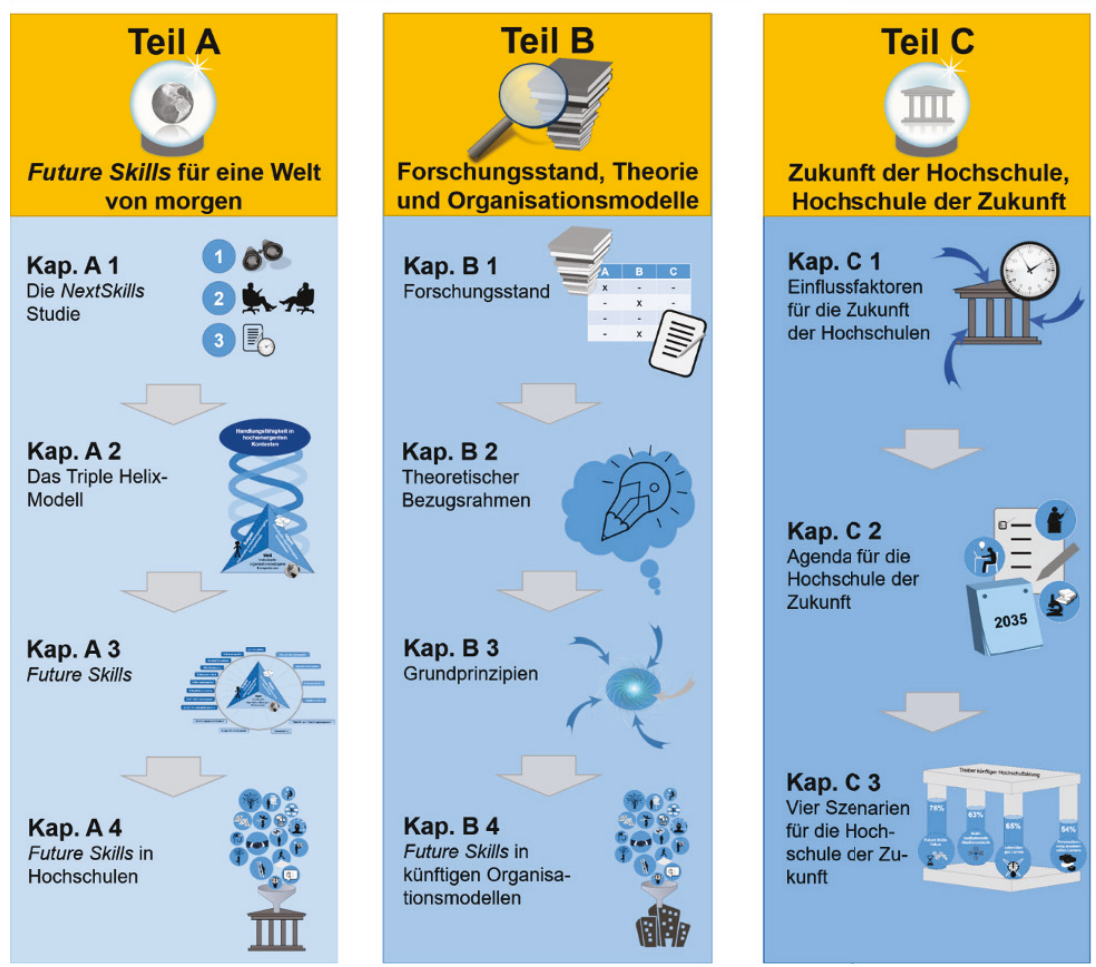

Abb. 1 Inhaltlicher Wegweiser: Struktur des Buches im Überblick 
Teil B des Buches widmet sich der Aufgabe, den Forschungsstand der Future Skills Forschung aufzuarbeiten. Dazu gibt es derzeit weder im deutschsprachigen noch im englischsprachigen Raum Vorlagen oder Literaturstudien. Kapitel B 1 stellt den Forschungsstand dar, angefangen bei der verwandten Forschung zum Thema Graduate Attributes. In Kapitel B2 wird der wesentliche theoretische Bezugsrahmen für die Future Skills Forschung konstruiert und beschrieben. Dabei spielt die sogenannte „Drift-to-Self Organisation“ eine besondere Rolle.

Es wird aufgearbeitet, welche Beiträge Bezugstheorien aus einem breiten interdisziplinären Spektrum zur Erklärung von Future Skills leisten. Hierfür werden theoretische Beiträge aus der Systemtheorie, Organisationstheorie, der Organisationssoziologie, der Managementtheorie, der Physik sowie der Bildungstheorie analysiert. Kapitel B 3 stellt Grundprinzipien dar, die der Konstruktion von Future Skills zugrunde liegen. Schließlich werden in Kapitel B 4 Organisationsmodelle analysiert, die die „Drift-to-Self-Organisation“ repräsentieren und deren Relevanz für die Bedeutung von Future Skills aufgezeigt.

Teil C des Buches widmet sich der Frage, wie die Hochschule der Zukunft aussehen wird. Zunächst werden in Kapitel C 1 die zehn wesentlichen Treiber beschrieben und analysiert, wie sie die Entwicklung der Hochschulen beeinflussen. In Kapitel C2 wird dann sowohl aus hochschuldidaktischer Perspektive als auch aus organisatorischer Perspektive beschrieben, wie Hochschulen sich auf Basis dieser Treiber entwickeln werden. Kapitel C 3 formuliert abschließend vier Szenarien für die Hochschule der Zukunft.

Das Buch entwickelt im Glossar ein wichtiges System von Querverweisen für die teilweise begrifflich aufwändige Arbeit. Zudem wird eine umfassende Bibliographie der deutsch- und englischsprachigen Literatur zum Thema dokumentiert. 
Open Access Dieses Kapitel wird unter der Creative Commons Namensnennung 4.0 International Lizenz (http://creativecommons.org/licenses/by/4.0/deed.de) veröffentlicht, welche die Nutzung, Vervielfältigung, Bearbeitung, Verbreitung und Wiedergabe in jeglichem Medium und Format erlaubt, sofern Sie den/die ursprünglichen Autor(en) und die Quelle ordnungsgemäß nennen, einen Link zur Creative Commons Lizenz beifügen und angeben, ob Änderungen vorgenommen wurden.

Die in diesem Kapitel enthaltenen Bilder und sonstiges Drittmaterial unterliegen ebenfalls der genannten Creative Commons Lizenz, sofern sich aus der Abbildungslegende nichts anderes ergibt. Sofern das betreffende Material nicht unter der genannten Creative Commons Lizenz steht und die betreffende Handlung nicht nach gesetzlichen Vorschriften erlaubt ist, ist für die oben aufgeführten Weiterverwendungen des Materials die Einwilligung des jeweiligen Rechteinhabers einzuholen.

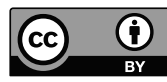

